\title{
Solving Differential Equations of Fractional Order Using Combined Adomian Decomposition Method with Kamal Integral Transformation
}

\author{
Muhamad Deni Johansyah ${ }^{1, *}$, Asep K Supriatna ${ }^{1}$, Endang Rusyaman $^{1}$, Jumadil Saputra $^{2}$ \\ ${ }^{1}$ Department of Mathematics, Faculty of Mathematics and Natural Sciences, Universitas Padjadjaran, Indonesia \\ ${ }^{2}$ School of Social and Economic Development, Universiti Malaysia Terengganu, Malaysia
}

Received November 2, 2021; Revised December 31, 2021; Accepted January 16, 2022

\section{Cite This Paper in the following Citation Styles}

(a): [1] Muhamad Deni Johansyah, Asep K Supriatna, Endang Rusyaman, Jumadil Saputra, "Solving Differential Equations of Fractional Order Using Combined Adomian Decomposition Method with Kamal Integral Transformation," Mathematics and Statistics, Vol. 10, No. 1, pp. 187 - 194, 2022. DOI: 10.13189/ms.2022.100117.

(b): Muhamad Deni Johansyah, Asep K Supriatna, Endang Rusyaman, Jumadil Saputra (2022). Solving Differential Equations of Fractional Order Using Combined Adomian Decomposition Method with Kamal Integral Transformation. Mathematics and Statistics, 10(1), 187 - 194. DOI: 10.13189/ms.2022.100117.

Copyright $\mathrm{C} 2022$ by authors, all rights reserved. Authors agree that this article remains permanently open access under the terms of the Creative Commons Attribution License 4.0 International License

\begin{abstract}
The differential equation is an equation that involves the derivative (derivatives) of the dependent variable with respect to the independent variable (variables). The derivative represents nothing but a rate of change, and the differential equation helps us present a relationship between the changing quantity with respect to the change in another quantity. The Adomian decomposition method is one of the iterative methods that can be used to solve differential equations, both integer and fractional order, linear or nonlinear, ordinary or partial. This method can be combined with integral transformations, such as Laplace, Sumudu, Natural, Elzaki, Mohand, Kashuri-Fundo, and Kamal. The main objective of this research is to solve differential equations of fractional order using a combination of the Adomian decomposition method with the Kamal integral transformation. Furthermore, the solution of the fractional differential equation using the combined method of the Adomian decomposition method and the Kamal integral transformation was investigated. The main finding of our study shows that the combined method of the Adomian decomposition method and the Kamal integral transformation is very accurate in solving differential equations of fractional order. The present results are original and new for solving differential equations of fractional order. The results attained in this paper confirm the illustrative example has been solved to show the efficiency of the proposed method.
\end{abstract}

Keywords Adomian Decomposition Method, Kamal Transformation, Fractional Differential Equation

Mathematics Subject Classification: 44A11; 65R10

\section{Introduction}

The Adomian decomposition method can be used to solve various problems in science and engineering [1]-[5]. The differential equations can be solved by this decomposition method can be in the form of integer or fractional order [6], ordinary or partial differential equations [7]-[8], initial value or boundary value problems [9]-[10], variable coefficient or constant [11]-[12], linear or nonlinear [13]-[14], homogeneous or nonhomogeneous [15]-[16]. The Adomian decomposition methods can be combined with integral transformations, such as Laplace [17], Sumudu [18], Natural [19], Elzaki [20], Mohand [21], and Kashuri-Fundo [22].

Some literature about Adomian decomposition method with Kamal integral transformation can be seen in [23]-[27]. Khandelwal et al. [23] studied solution of Blasius differential equation with condition at infinity and converted the series solution into rational function by using

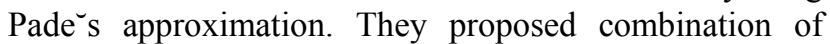
Adomian decomposition method and Kamal transform for 
handling a differential equation of mixing layer that arises in viscous incompressible fluid. When the exact solution has a closed form, Ahmad [24] proposed a new method for solving nonlinear wave-like equations with variable coefficients. They discovered that the Kamal decomposition method is both efficient and easy, and that it may be used to solve a variety of nonlinear problems. Khandelwal et al. [25] solve coupled system of non- linear partial differential equations by the Kamal transform and the Adomian decomposition method. With this combined, they found that the exact solution of linear and non-linear partial differential equations. Pushpam and Kumar [26] solved nonlinear delay differential equations using Kamal transform and Adomian decomposition method. They validated the nonlinear delay differential equations using numerical simulations to illustrate the accurateness of the proposed method. Ahmad and Ayub [27] solve analytically the integro-differential equations using modified Kamal decomposition method using Adomian polynomials. They show that the proposed method very powerful, efficient, and simple and can be applied to linear and nonlinear problems. However, there has been little discussion on combined of Adomian decomposition method with Kamal integral transformation.

Motivated by the combined of Adomian decomposition method with Kamal integral transformation, the aim of our work is to broaden the knowledge of differential equations of fractional order with Adomian decomposition method and Kamal integral transformation. Furthermore, the solution of the fractional differential equation using the combined method of the Adomian decomposition method and the Kamal integral transformation was investigated.

The Combined Method of the Adomian Decomposition Method and the Kamal Integral Transformation is very accurate in solving fractional order differential equations and easier to determine the terms of the fractional differential equation approximation solution

The rest of the paper is organized as follows. The basic theory of the Adomian decomposition method and the Kamal integral transformation is presented in Section 2. In Section 3, the numerical solution of Adomian decomposition method and the Kamal integral transformation are demonstrated. The Conclusion is presented in Section 4.

\section{Basic Theory}

\subsection{Kamal Transformation}

Definition 2.1 [28].

Given a set of functions

$$
\begin{gathered}
S=\left\{f(x): \exists M, k_{1}, k_{2}>0,|f(x)|<M e^{\frac{|x|}{k_{j}}}, x\right. \\
\left.\in(-1)^{j} \times[0, \infty)\right\},
\end{gathered}
$$

The Kamal's transformation is defined as

$$
\mathcal{G}[f(x)]=G(v)=\int_{0}^{\infty} f(x) e^{-\frac{x}{v}} d x, x \geq 0, k_{1} \leq v \leq k_{2} .
$$

Furthermore, the inverse of the Kamal transformation is denoted by $\mathcal{G}^{-1}[G(v)]=f(x), x \geq 0$.

The following are some cases of Kamal transformations for the following simple functions:

$$
\begin{gathered}
f(x)=1 \text { where } x \geq 0 \text {, based on definition 2.1, we get } \\
\mathcal{G}[f(x)]=G(v)=\int_{0}^{\infty} f(x) e^{-\frac{x}{v}} d x, x \geq 0, k_{1} \leq v \leq k_{2} . \\
\mathcal{G}[1]=G(v)=\int_{0}^{\infty} e^{-\frac{x}{v}} d x=\left[-v e^{-\frac{x}{v}}\right]_{0}^{\infty}=v .
\end{gathered}
$$

- $f(x)=x$ where $x \geq 0$, based on definition 2.1 and using partial integrals, we get

$$
\begin{gathered}
\mathcal{G}[f(x)]=G(v)=\int_{0}^{\infty} f(x) e^{-\frac{x}{v}} d x, x \geq 0, k_{1} \leq v \leq k_{2} . \\
\mathcal{G}[x]=G(v)=\int_{0}^{\infty} x e^{-\frac{x}{v}} d x=v^{2} .
\end{gathered}
$$

For $f(x)=x^{n}$ where $x \geq 0$, we get

$$
\mathcal{G}\left[x^{n}\right]=n ! v^{n+1} .
$$

Based on that, for $n=\alpha$, where $\alpha$ is a fractional number, then applies

$$
\mathcal{G}\left[x^{\alpha}\right]=\Gamma(\alpha+1) v^{\alpha+1}
$$

Definition 2.2 [29].

The Caputo fractional derivative of the function $f$ with respect to $x$ of the order $\alpha$, where $\alpha>0$, is defined as

$$
\begin{gathered}
{ }_{a}^{C} D_{x}^{\alpha} f(x)=\frac{1}{\Gamma(n-\alpha)} \int_{a}^{x}(x-u)^{n-\alpha-1} f^{(n)}(u) d u, n-1 \\
<\alpha \leq n .
\end{gathered}
$$

Theorem 2.1 [28]

Let $G(v)$ be the Kamal transformation of $f(x)$, then

$$
\begin{aligned}
& \text { - } G\left[f^{\prime}(x)\right]=\frac{1}{v} G(v)-f(0), \\
& \text { - } G\left[f^{\prime \prime}(x)\right]=\frac{1}{v^{2}} G(v)-\frac{1}{v} f(0)-f^{\prime}(0), \\
& \text { - } G\left[f^{(n)}(x)\right]=\frac{1}{v^{n}} G(v)-\sum_{k=0}^{n-1} \frac{f^{(k)}(0)}{v^{n-k-1}}
\end{aligned}
$$

Theorem 2.2 [28]

The Kamal transformation of the Caputo fractional derivative for $\boldsymbol{\alpha}=0$ is defined as

$$
\mathcal{G}\left[{ }^{C} D_{x}^{\alpha} f(x)\right]=\frac{G(v)}{v^{\alpha}}-\sum_{k=0}^{n-1} \frac{f^{(k)}(0)}{v^{\alpha-k-1}}, n-1<\alpha \leq n .
$$

\subsection{Combined Method}

The combined method is the method obtained from the combination of the Adomian decomposition method with the "Kamal" integral transformation. This section presents solutions to fractional differential equations using the 
combined method.

Given the fractional differential equation as follows

$$
D_{x}^{\alpha} y(x)=g(x)+N y(x)+R y(x)
$$

with the initial condition $y(0)=c$, where $\boldsymbol{D}_{x}^{\alpha} \equiv{ }^{C} \boldsymbol{D}_{x}^{\alpha}$ is the Caputo fractional derivative operator and $\mathbf{0}<\boldsymbol{\alpha} \leq \mathbf{1}$. Furthermore, equation (2) is transformed using the Kamal transformation, we get

$$
\begin{gathered}
\mathcal{G}\left[D_{x}^{\alpha} y(x)\right]=\mathcal{G}[g(x)+N y(x)+R y(x)], \\
\mathcal{G}\left[{ }^{C} D_{x}^{\alpha} f(x)\right]=\frac{G(v)}{v^{\alpha}}-\sum_{k=0}^{n-1} \frac{f^{(k)}(0)}{v^{\alpha-k-1}}, \\
n-1<\alpha \leq n . \\
\frac{y(v)}{v^{\alpha}}-\frac{y(0)}{v^{\alpha-1}}=\mathcal{G}[g(x)]+\mathcal{G}[N y(x)]+\mathcal{G}[R y(x)], \\
y(v)-v y(0)=v^{\alpha} \mathcal{G}[g(x)]+v^{\alpha} \mathcal{G}[N y(x)] \\
+v^{\alpha} \mathcal{G}[R y(x)], \\
y(v)=v y(0)+v^{\alpha} \mathcal{G}[g(x)]+v^{\alpha} \mathcal{G}[N y(x)] \\
+v^{\alpha} \mathcal{G}[R y(x)] .
\end{gathered}
$$

Furthermore, using the inverse of the Kamal transform: $\mathcal{G}^{-1}[\boldsymbol{y}(\boldsymbol{v})]=\boldsymbol{y}(\boldsymbol{x})$ and $\mathcal{G}^{-\mathbf{1}}[\boldsymbol{v}]=\mathbf{1}$, in equation (3), we get

$$
\begin{aligned}
\mathcal{G}^{-1}[y(v)] & =\mathcal{G}^{-1}\left[v y(0)+v^{\alpha} \mathcal{G}[g(x)]\right. \\
& \left.+v^{\alpha} \mathcal{G}[N y(x)]+v^{\alpha} \mathcal{G}[R y(x)]\right], \\
y(x)= & y(0)+\mathcal{G}^{-1}\left[v^{\alpha} \mathcal{G}[g(x)]\right] \\
& +\mathcal{G}^{-1}\left[v^{\alpha} \mathcal{G}[N y(x)]\right] \\
& +\mathcal{G}^{-1}\left[v^{\alpha} \mathcal{G}[R y(x)]\right] .
\end{aligned}
$$

The Adomian decomposition method assumes that the function $y$ can be decomposed into an infinite series as follows

$$
y(x)=\sum_{n=0}^{\infty} y_{n}(x),
$$

where $y_{\mathrm{n}}$ can be determined recursively. This method also assumes that the nonlinear operator $N_{\mathrm{y}}$ can be decomposed into an infinite polynomial series as follows

$$
y(x)=\sum_{n=0}^{\infty} y_{n}(x)
$$

where $A_{\mathrm{n}}$ is an Adomian polynomial, defined as

$$
A_{n}=\frac{1}{n !} \frac{d^{n}}{d \lambda^{n}}\left[N\left(\sum_{k=0}^{n} \lambda^{k} w_{k}\right)\right]_{\lambda=0}, \quad n=0,1,2, \ldots
$$

where $\lambda$ is a parameter. Adomian polynomial $A_{\mathrm{n}}$ can be described as follows

$$
\begin{gathered}
A_{0}=\frac{1}{0 !} \frac{d^{0}}{d \lambda^{0}}\left[N\left(\sum_{k=0}^{0} \lambda^{k} y_{k}\right)\right]_{\lambda=0}=N\left(y_{0}\right) \\
A_{1}=\frac{1}{1 !} \frac{d^{1}}{d \lambda^{1}}\left[N\left(\sum_{k=0}^{1} \lambda^{k} y_{k}\right)\right]_{\lambda=0}=y_{1} N^{\prime}\left(y_{0}\right)
\end{gathered}
$$

$$
\begin{aligned}
A_{2}= & \frac{1}{2 !} \frac{d^{2}}{d \lambda^{2}}\left[N\left(\sum_{k=0}^{2} \lambda^{k} y_{k}\right)\right]_{\lambda=0} \\
& =y_{2} N^{\prime}\left(y_{0}\right)+\frac{y_{1}^{2}}{2 !} N^{\prime \prime}\left(y_{0}\right),
\end{aligned}
$$

Substituting equations (5) and (6) into equation (4), we get

$$
\begin{aligned}
\sum_{n=0}^{\infty} y_{n}(x)=y(0) & +\mathcal{G}^{-1}\left[v^{\alpha} \mathcal{G}[g(x)]\right]+\mathcal{G}^{-1}\left[v^{\alpha} \mathcal{G}\left[\sum_{n=0}^{\infty} A_{n}\right]\right] \\
& +\mathcal{G}^{-1}\left[v^{\alpha} \mathcal{G}\left[R \sum_{n=0}^{\infty} y_{n}(x)\right]\right] .
\end{aligned}
$$

So, the recursive relation is obtained from the solution of the fractional differential equation using the Kamal decomposition method (2) as follows

$$
\begin{gathered}
y_{0}=y(0)+\mathcal{G}^{-1}\left[v^{\alpha} \mathcal{G}[g(x)]\right], \\
y_{n+1}=\mathcal{G}^{-1}\left[v^{\alpha} \mathcal{G}\left[A_{n}\right]\right]+\mathcal{G}^{-1}\left[v^{\alpha} \mathcal{K}\left[R y_{n}\right]\right],
\end{gathered}
$$

\section{Result and Discussion}

This section presents some numerical examples of solving fractional differential equations using the Kamal decomposition method.

\section{Example 3.1}

Given the fractional differential equation as follows

$$
D_{x}^{\alpha} y(x)=y^{2}(x)+1, x>0,0<\alpha \leq 1,
$$

with the initial conditions $y(0)=0$, and the exact solution, i.e.

$$
y(x)=\tan (x) .
$$

Based on the recursive formula in equation (8), the approximate solution of the fractional differential equation (9) uses the combined method of the Adomian decomposition method and the Kamal integral transformation as follows:

$$
\begin{gathered}
y_{0}=\mathcal{G}^{-1}\left[v^{\alpha} \mathcal{G}[1]\right], \\
y_{n+1}=\mathcal{G}^{-1}\left[v^{\alpha} \mathcal{G}\left[A_{n}\right]\right], \quad n=0,1,2, \ldots .
\end{gathered}
$$

where $A_{\mathrm{n}}$ is the Adomian polynomial of the nonlinear operator $N y=y^{2}$, which can be described as follows

$$
\begin{gathered}
A_{0}=y_{0}^{2}, \\
A_{1}=2 y_{0} y_{1}, \\
A_{2}=2 y_{0} y_{2}+y_{1}^{2},
\end{gathered}
$$

The following is a description of the solution to the equation (11):

$$
y_{0}=\mathcal{G}^{-1}\left[v^{\alpha} \mathcal{G}[1]\right]=\mathcal{G}^{-1}\left[v^{\alpha} v\right]=\mathcal{G}^{-1}\left[v^{\alpha+1}\right]=\frac{x^{\alpha}}{\Gamma(\alpha+1)^{\prime}}
$$




$$
\begin{aligned}
& y_{1}=\mathcal{G}^{-1}\left[v^{\alpha} \mathcal{G}\left[A_{0}\right]\right]=\mathcal{G}^{-1}\left[v^{\alpha} \mathcal{G}\left[y_{0}^{2}\right]\right] \\
& =\mathcal{G}^{-1}\left[v^{\alpha} \mathcal{G}\left[\frac{x^{2 \alpha}}{\Gamma^{2}(\alpha+1)}\right]\right] \\
& =\mathcal{G}^{-1}\left[v^{\alpha} \frac{\Gamma(2 \alpha+1)}{\Gamma^{2}(\alpha+1)} v^{2 \alpha+1}\right] \\
& =\mathcal{G}^{-1}\left[\frac{\Gamma(2 \alpha+1)}{\Gamma^{2}(\alpha+1)} v^{3 \alpha+1}\right] \\
& =\frac{\Gamma(2 \alpha+1)}{\Gamma^{2}(\alpha+1) \Gamma(3 \alpha+1)} x^{3 \alpha}, \\
& \begin{array}{r}
y_{2}=\mathcal{G}^{-1}\left[v^{\alpha} \mathcal{G}\left[A_{1}\right]\right]=\mathcal{G}^{-1}\left[v^{\alpha} \mathcal{G}\left[2 y_{0} y_{1}\right]\right] \\
=\mathcal{G}^{-1}\left[v^{\alpha} \mathcal{G}\left[2 \frac{x^{\alpha}}{\Gamma(\alpha+1)} \frac{\Gamma(2 \alpha+1)}{\Gamma^{2}(\alpha+1) \Gamma(3 \alpha+1)} x^{3 \alpha}\right]\right]
\end{array} \\
& =\mathcal{G}^{-1}\left[v^{\alpha} \mathcal{G}\left[\frac{2 \Gamma(2 \alpha+1)}{\Gamma^{3}(\alpha+1) \Gamma(3 \alpha+1)} x^{4 \alpha}\right]\right] \\
& =\mathcal{G}^{-1}\left[v^{\alpha} \frac{2 \Gamma(2 \alpha+1) \Gamma(4 \alpha+1)}{\Gamma^{3}(\alpha+1) \Gamma(3 \alpha+1)} v^{4 \alpha+1}\right] \\
& =\mathcal{G}^{-1}\left[\frac{2 \Gamma(2 \alpha+1) \Gamma(4 \alpha+1)}{\Gamma^{3}(\alpha+1) \Gamma(3 \alpha+1)} v^{5 \alpha+1}\right] \\
& =\frac{2 \Gamma(2 \alpha+1) \Gamma(4 \alpha+1)}{\Gamma^{3}(\alpha+1) \Gamma(3 \alpha+1) \Gamma(5 \alpha+1)} x^{5 \alpha}, \\
& y(x)=y_{0}+y_{1}+y_{2}+\cdots \\
& =\frac{x^{\alpha}}{\Gamma(\alpha+1)}+\frac{\Gamma(2 \alpha+1)}{\Gamma^{2}(\alpha+1) \Gamma(3 \alpha+1)} x^{3 \alpha} \\
& +\frac{2 \Gamma(2 \alpha+1) \Gamma(4 \alpha+1)}{\Gamma^{3}(\alpha+1) \Gamma(3 \alpha+1) \Gamma(5 \alpha+1)} x^{5 \alpha}
\end{aligned}
$$

Figure 1 shows the approximate solution of equation (9) to the tenth iteration of the fractional differential equation using the Combined Method (11) for different $\alpha$ values, namely $\alpha=0.9, \alpha=0.99, \alpha=0.999$ and $\alpha=1$ with the help of MAPLE software.

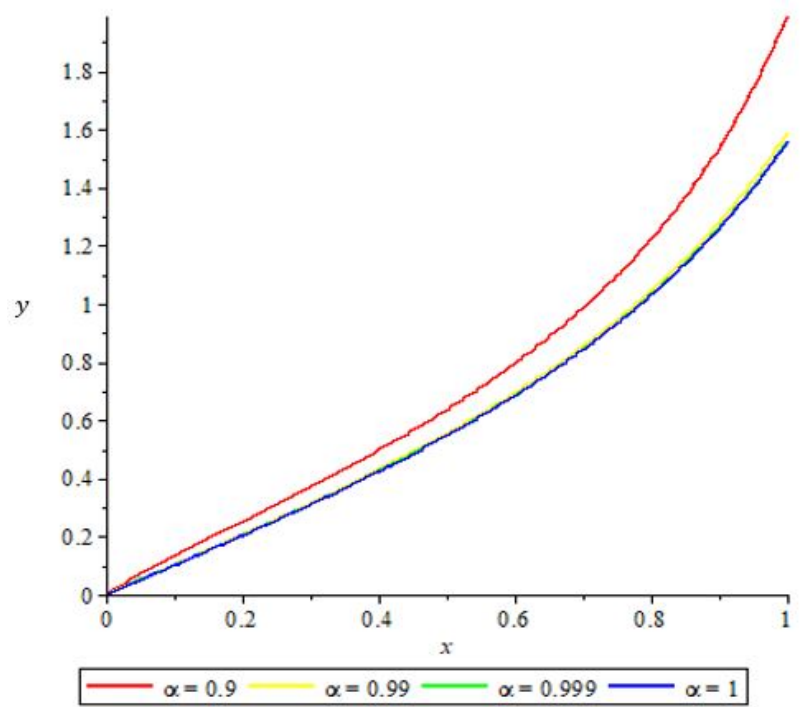

Figure 1. The graph of the solution of the fractional differential equation using the Combined Method of the Adomian Decomposition Method and the Kamal Integral Transform (11) for $\alpha=0.9, \alpha=0.99, \alpha=0.999$ and $\alpha=1$

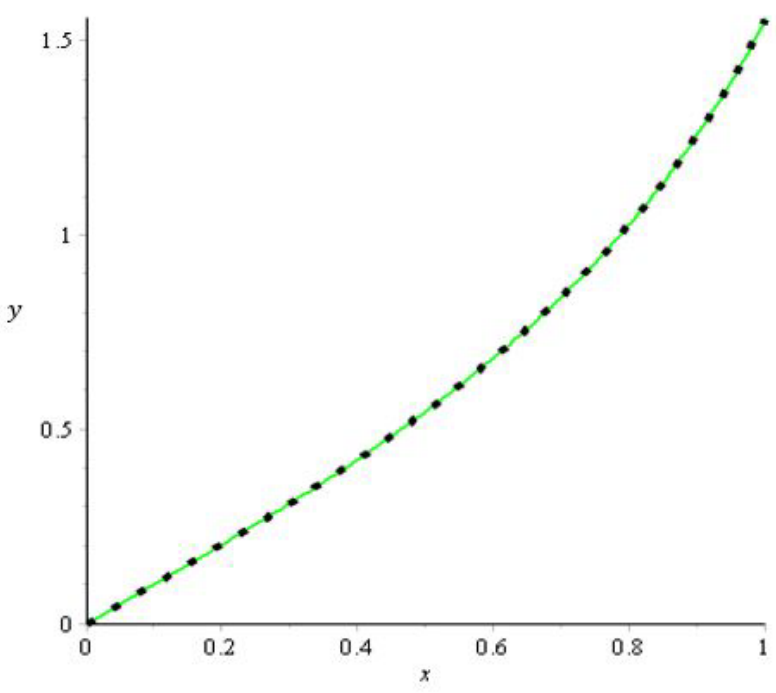

Figure 2. The graph of the exact solution (green curve) coincides with the approximation solution using the Combined Method for $\alpha=1$ (black dot curve)

Furthermore, Figure 2 shows a graph of the exact solution of equation (10) the green curve coincides with the approximation solution using the Combined Method (11) the black dot curve for $\alpha=1$ with the help of MAPLE software.

Based on Figure 2, it can be seen that the graph of the exact solution of equation (10) green colored curve coincides with the black dot approach solution graph of equation (11) for $\alpha=1$. This shows that the Combined Method of the Adomian Decomposition Method and the Kamal Integral Transformation is very accurate in solving differential equations of fractional order.

\section{Example 3.2}

Given the following fractional differential equation [30];

$$
D_{x}^{\alpha} y(x)=1-y^{2}(x), x>0,0<\alpha \leq 1,
$$

with initial conditions is $w(0)=0$, exact solution, i.e.

$$
y(x)=\frac{e^{2 x}-1}{e^{2 x}+1}
$$

Draw a graph of the solution of the Riccati fractional differential equation $y(x)$ using the combined method for $\alpha=0.7 ; 0.8 ; 0.9$ and 1 . Does the graph of the function $\mathrm{y}(\mathrm{x})$ use the Combined Method for $\alpha=1$ coincide with the exact solution?

Based on the recursive formula in equation (7), the approximate solution of the fractional differential equation (12) uses the Combined Method of the Adomian Decomposition Method and the Kamal Integral Transformation as follows

$$
\begin{aligned}
y_{0} & =\mathcal{G}^{-1}\left[v^{\alpha} \mathcal{G}[1]\right], \\
y_{n+1} & =-\mathcal{G}^{-1}\left[v^{\alpha} \mathcal{G}\left[A_{n}\right]\right], n=0,1,2, \ldots .
\end{aligned}
$$

where $A_{n}$ is Adomian polynomial of the nonlinear operator $N y=y^{2}$, which can be described as follows 


$$
\begin{aligned}
& A_{0}=y_{0}^{2} \\
& A_{1}=2 y_{0} y_{1} \\
& A_{2}=2 y_{0} y_{2}+y_{1}^{2},
\end{aligned}
$$

The following describes the solution approach (14):

$$
\begin{aligned}
& y_{0}=\mathcal{G}^{-1}\left[v^{\alpha} \mathcal{G}[1]\right]=\mathcal{G}^{-1}\left[v^{\alpha} v\right]=\mathcal{G}^{-1}\left[v^{\alpha+1}\right] \\
& =\frac{x^{\alpha}}{\Gamma(\alpha+1)} \\
& y_{1}=-\mathcal{G}^{-1}\left[v^{\alpha} \mathcal{G}\left[A_{0}\right]\right]=-\mathcal{G}^{-1}\left[v^{\alpha} \mathcal{G}\left[y_{0}^{2}\right]\right] \\
& =-\mathcal{G}^{-1}\left[v^{\alpha} \mathcal{G}\left[\frac{x^{2 \alpha}}{\Gamma^{2}(\alpha+1)}\right]\right] \\
& =-\mathcal{G}^{-1}\left[v^{\alpha} \frac{\Gamma(2 \alpha+1)}{\Gamma^{2}(\alpha+1)} v^{2 \alpha+1}\right] \\
& =-\mathcal{G}^{-1}\left[\frac{\Gamma(2 \alpha+1)}{\Gamma^{2}(\alpha+1)} v^{3 \alpha+1}\right] \\
& =-\frac{\Gamma(2 \alpha+1)}{\Gamma^{2}(\alpha+1) \Gamma(3 \alpha+1)} x^{3 \alpha} \\
& y_{2}=-\mathcal{G}^{-1}\left[v^{\alpha} \mathcal{G}\left[A_{1}\right]\right]=-\mathcal{G}^{-1}\left[v^{\alpha} \mathcal{G}\left[2 y_{0} y_{1}\right]\right] \\
& =-\mathcal{G}^{-1}\left[v^{\alpha} \mathcal{G}\left[2 \frac{x^{\alpha}}{\Gamma(\alpha+1)}\left(-\frac{\Gamma(2 \alpha+1)}{\Gamma^{2}(\alpha+1) \Gamma(3 \alpha+1)} x^{3 \alpha}\right)\right]\right] \\
& =\mathcal{G}^{-1}\left[v^{\alpha} \mathcal{G}\left[\frac{2 \Gamma(2 \alpha+1)}{\Gamma^{3}(\alpha+1) \Gamma(3 \alpha+1)} x^{4 \alpha}\right]\right] \\
& =\mathcal{G}^{-1}\left[v^{\alpha} \frac{2 \Gamma(2 \alpha+1) \Gamma(4 \alpha+1)}{\Gamma^{3}(\alpha+1) \Gamma(3 \alpha+1)} v^{4 \alpha+1}\right] \\
& =\mathcal{G}^{-1}\left[\frac{2 \Gamma(2 \alpha+1) \Gamma(4 \alpha+1)}{\Gamma^{3}(\alpha+1) \Gamma(3 \alpha+1)} v^{5 \alpha+1}\right] \\
& =\frac{2 \Gamma(2 \alpha+1) \Gamma(4 \alpha+1)}{\Gamma^{3}(\alpha+1) \Gamma(3 \alpha+1) \Gamma(5 \alpha+1)} x^{5 \alpha} \\
& y(x)=y_{0}+y_{1}+y_{2}+\cdots \\
& =\frac{x^{\alpha}}{\Gamma(\alpha+1)}-\frac{\Gamma(2 \alpha+1)}{\Gamma^{2}(\alpha+1) \Gamma(3 \alpha+1)} x^{3 \alpha} \\
& +\frac{2 \Gamma(2 \alpha+1) \Gamma(4 \alpha+1)}{\Gamma^{3}(\alpha+1) \Gamma(3 \alpha+1) \Gamma(5 \alpha+1)} x^{5 \alpha} \\
& +\cdots
\end{aligned}
$$

Figure 3 shows a graph of the approximate solution of equation (12) to the tenth iteration of the fractional differential equation using the Combined Method (14) for values $\alpha$ which are different, namely $\alpha=0.7, \alpha=$ $0.8, \alpha=0.9$ and $\alpha=1$ with the help of Maple software.

Furthermore, Figure 4 shows a graph of the exact solution of equation (13) the green-colored curve coincides with the approximation solution using the Combined Method (14) with black dots for $\alpha=1$ with the help of Maple software.

Based on Figure 4, it can be seen that the graph of the exact solution of equation (13) green colored curve coincides with the black dot approach solution graph of equation (14) for $\alpha=1$. This shows that the Combined Method of the Adomian Decomposition Method and the Kamal Integral Transformation is very accurate in solving differential equations of fractional order.

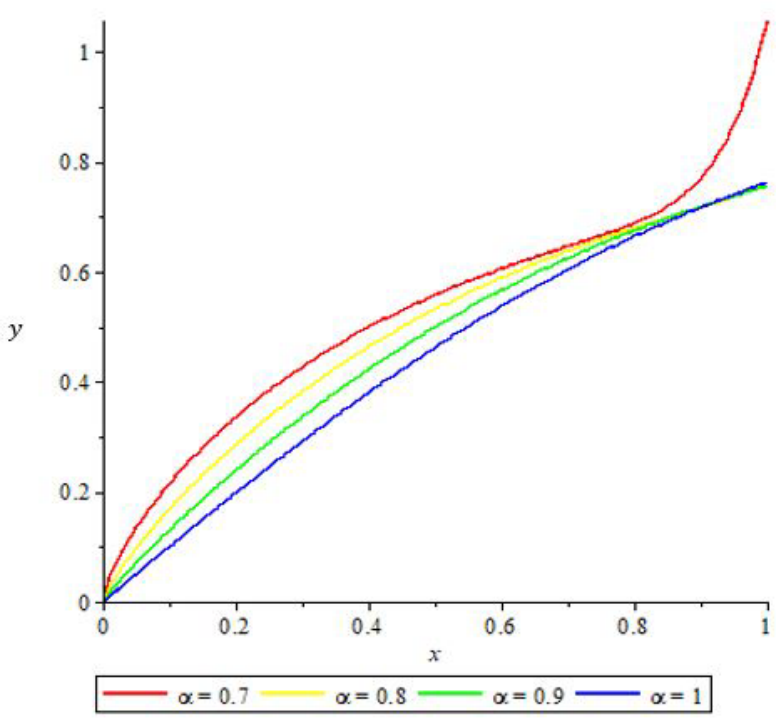

Figure 3. The graph of the solution to the fractional differential equation using the Combined Method of the Adomian Decomposition Method and the Kamal Integral Transform (14) for $\alpha=0.7, \alpha=0.8, \alpha=$ 0.9 and $\alpha=1$

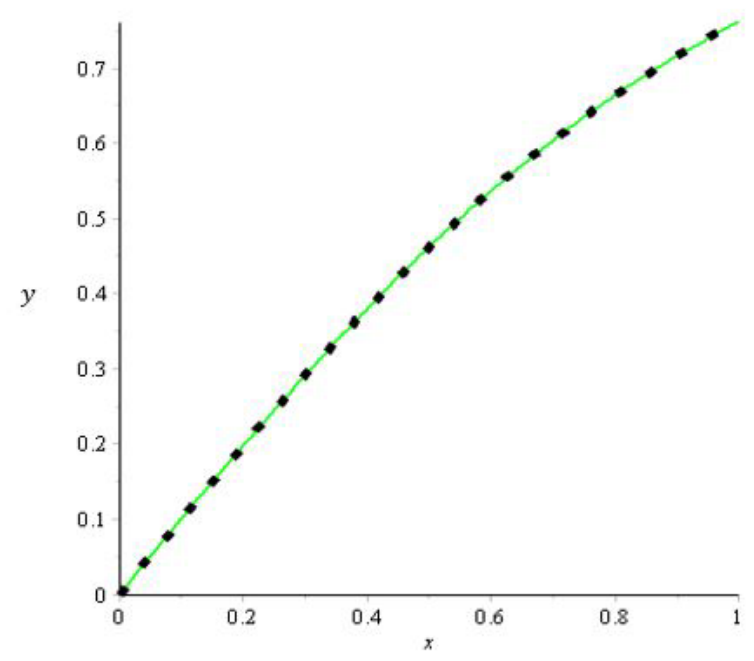

Figure 4. The graph of the exact solution (green line) coincides with the approximation solution using the Combined Method for $\alpha=1$.

\section{Example 3.3}

Given the following fractional differential equation [31].

$$
D_{x}^{\alpha} y(x)=-y(x)+y^{2}(x), x>0,0<\alpha \leq 1,
$$

with initial conditions is $y(0)=\frac{1}{2}$, exact solution, i.e.

$$
y(x)=\frac{e^{-x}}{1+e^{-x}}
$$

Draw a graph of the solution of the Riccati fractional differential equation $y(x)$ using the combined method for $\alpha=0.7 ; 0.8 ; 0.9$ and 1 . Does the graph of the function $\mathrm{y}(\mathrm{x})$ using the Combined Method for $\alpha=1$ coincide with the exact solution?

Based on the recursive formula in equation (7), the approximate solution of the fractional differential equation 
(15) uses the Combined Method of the Adomian Decomposition Method and the Kamal Integral Transformation as follows

$$
\begin{aligned}
y_{0} & =\frac{1}{2}, \\
y_{n+1} & =-\mathcal{G}^{-1}\left[v^{\alpha} \mathcal{G}\left[R_{n}\right]\right]+\mathcal{G}^{-1}\left[v^{\alpha} \mathcal{G}\left[A_{n}\right]\right], n \\
& =0,1,2, \ldots .
\end{aligned}
$$

where $A_{n}$ is Adomian polynomial of the nonlinear operator $N y=y^{2}$, which can be described as follows [32]-[33]

$$
\begin{aligned}
& A_{0}=y_{0}^{2}, \\
& A_{1}=2 y_{0} y_{1}, \\
& A_{2}=2 y_{0} y_{2}+y_{1}^{2},
\end{aligned}
$$

The following describes the solution approach (17):

$$
\begin{gathered}
y_{0}=\frac{1}{2} \\
y_{1}=-\mathcal{G}^{-1}\left[v^{\alpha} \mathcal{G}\left[R_{0}\right]\right]+\mathcal{G}^{-1}\left[v^{\alpha} \mathcal{G}\left[A_{0}\right]\right] \\
=-\mathcal{G}^{-1}\left[v^{\alpha} \mathcal{G}\left[y_{0}\right]\right]+\mathcal{G}^{-1}\left[v^{\alpha} \mathcal{G}\left[y_{0}^{2}\right]\right] \\
=-\mathcal{G}^{-1}\left[v^{\alpha} \mathcal{G}\left[\frac{1}{2}\right]\right]+\mathcal{G}^{-1}\left[v^{\alpha} \mathcal{G}\left[\frac{1}{4}\right]\right] \\
=-\mathcal{G}^{-1}\left[v^{\alpha} \mathcal{G}\left[\frac{1}{4}\right]\right]=-\mathcal{G}^{-1}\left[\frac{1}{4} v^{\alpha} v\right] \\
=-\mathcal{G}^{-1}\left[\frac{1}{4} v^{\alpha+1}\right]=-\frac{1}{4 \Gamma(\alpha+1)} x^{\alpha} \\
y_{2}=-\mathcal{G}^{-1}\left[v^{\alpha} \mathcal{G}\left[R_{1}\right]\right]+\mathcal{G}^{-1}\left[v^{\alpha} \mathcal{G}\left[A_{1}\right]\right] \\
=-\mathcal{G}^{-1}\left[v^{\alpha} \mathcal{G}\left[y_{1}\right]\right]+\mathcal{G}^{-1}\left[v^{\alpha} \mathcal{G}\left[2 y_{0} y_{1}\right]\right] \\
=-\mathcal{G}^{-1}\left[v^{\alpha} \mathcal{G}\left[-\frac{1}{4 \Gamma(\alpha+1)} x^{\alpha}\right]\right] \\
+\mathcal{G}^{-1}\left[v^{\alpha} \mathcal{G}\left[2 \frac{1}{2}\left(-\frac{1}{4 \Gamma(\alpha+1)} x^{\alpha}\right)\right]\right] \\
=\mathcal{G}^{-1}\left[v^{\alpha} \mathcal{G}\left[\frac{1}{4 \Gamma(\alpha+1)} x^{\alpha}\right]\right] \\
-\mathcal{G}^{-1}\left[v^{\alpha} \mathcal{G}\left[\frac{1}{4 \Gamma(\alpha+1)} x^{\alpha}\right]=0\right. \\
y(x)=y_{0}+y_{1}+y_{2}+\cdots=\frac{1}{2}-\frac{1}{4 \Gamma(\alpha+1)} x^{\alpha}+0+\cdots
\end{gathered}
$$

Figure 5 shows a graph of the approximate solution of equation (15) to the tenth iteration of the fractional differential equation using the Combined Method (17) for values $\alpha$ which are different, namely $\alpha=0.7, \alpha=$ $0.8, \alpha=0.9$ and $\alpha=1$ with the help of Maple software.

Furthermore, Figure 6 shows a graph of the exact solution of equation (16) the green-colored curve coincides with the approximation solution using the Combined Method (17) with black dots for $\alpha=1$ with the help of Maple software.

Based on Figure 6, it can be seen that the graph of the exact solution of equation (16) green colored curve coincides with the black dot approach solution graph of equation (17) for $\alpha=1$. This shows that the Combined Method of the Adomian Decomposition Method and the
Kamal Integral Transformation is very accurate in solving differential equations of fractional order.

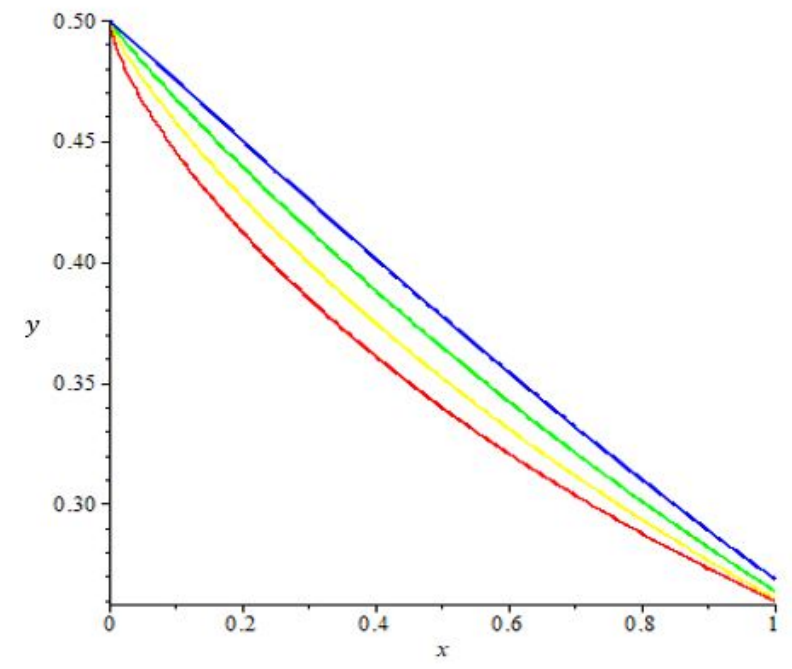

Figure 5. The graph of the solution to the fractional differential equation using the Combined Method of the Adomian Decomposition Method and the Kamal Integral Transform (17) for $\alpha=0.7, \alpha=0.8, \alpha=0.9$ and $\alpha=1$

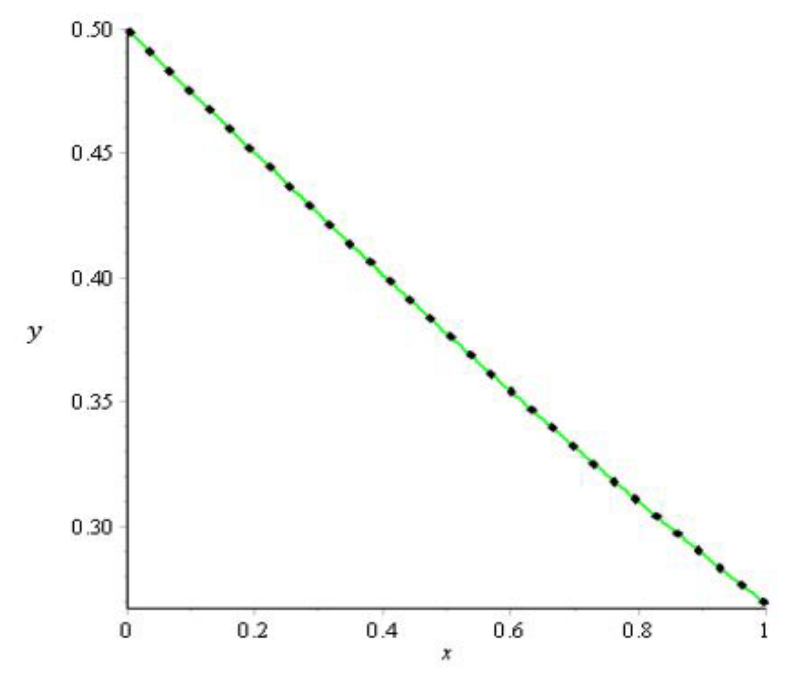

Figure 6. The graph of the exact solution (green line) coincides with the approximation solution using the Combined Method for $\alpha=1$

\section{Conclusions}

In this work, we report solve differential equations of fractional order using a combination of the Adomian decomposition method with the Kamal integral transformation. Furthermore, the solution of the fractional differential equation using the combined method of the Adomian decomposition method and the Kamal integral transformation was investigated. The main finding of our study shows that the combined method of the Adomian decomposition method and the Kamal integral transformation is very accurate, simple and can be applied to solve differential equations of fractional order and easier 
to determine the terms of the fractional differential equation approximation solution.

\section{Acknowledgements}

The authors would like to thank to the Rector of Universitas Padjadjaran for the project financial support through "Hibah Riset dan Teknologi" with contract number 1207/UN6.3.1/PT.00/2021

\section{Conflict of Interest}

There is no conflict of interest as declared by the authors.

\section{REFERENCES}

[1] Sambas, S. Vaidyanathan, E. Tlelo-Cuautle, S. Zhang, O. Guillen-Fernandez, Y. Hidayat, G. Gundara. A novel chaotic system with two circles of equilibrium points: multistability, electronic circuit and FPGA realization. Electronics, 8(11), 1211. https://doi.org/10.3390/electronic s8111211

[2] J. S. Duan, R. Rach, A. M. Wazwaz. Solution of the model of beam-type micro-and nano-scale electrostatic actuators by a new modified Adomian decomposition method for nonlinear boundary value problems. International Journal of Non-Linear Mechanics, 2013, Vol. 49, 159-169. https://doi.org/10.1016/j.ijnonlinmec.2012.10.003

[3] P. Y. Tsai, C. O. K. Chen. Free vibration of the nonlinear pendulum using hybrid Laplace Adomian decomposition method. International journal for numerical methods in biomedical engineering, 2011, Vol. 27, No. 2, 262-272. https://doi.org/10.1002/cnm.1304

[4] Sambas, S. Vaidyanathan, E. Tlelo-Cuautle, B. Abd-El-Atty, A. A. Abd El-Latif, O. Guillén-Fernández, O, Y. Hidayat, G. Gundara. A 3-D multi-stable system with a peanut-shaped equilibrium curve: Circuit design, FPGA realization, and an application to image encryption. IEEE Access, 2020, Vol. 8, 137116-137132. https://doi.org/10.1 109/ACCESS.2020.3011724

[5] Sambas, S. Vaidyanathan, T. Bonny, S. Zhang, Y. Hidayat, G. Gundara, M. Mamat. Mathematical Model and FPGA Realization of a Multi-Stable Chaotic Dynamical System with a Closed Butterfly-Like Curve of Equilibrium Points. Applied Sciences, 2021, Vol. 11, No. 2, 788. https://doi.or g/10.3390/app11020788

[6] G. C. Wu, D. Baleanu. Variational iteration method for the Burgers' flow with fractional derivatives - new Lagrange multipliers. Applied Mathematical Modelling, 2013, Vol. 37, No. 9, 6183-6190. https://doi.org/10.1016/j.apm.2012. 12.018

[7] N. Bildik, A. Konuralp. The use of variational iteration method, differential transform method and Adomian decomposition method for solving different types of nonlinear partial differential equations. International Journal of Nonlinear Sciences and Numerical Simulation, 2006, Vol. 7, No. 1, 65-70. https://doi.org/10.1515/IJNSN S.2006.7.1.65

[8] G. Nhawu, P. Mafuta, J. Mushanyu. The Adomian decomposition method for numerical solution of first-order differential equations. J. Math. Comput. Sci., 2016, Vol. 6, No. 3, 307-314. http://www.scik.org/index.php/jmcs/articl e/view/2469

[9] M. Tatari, M. Dehghan. The use of the Adomian decomposition method for solving multipoint boundary value problems. Physica Scripta, 2006, Vol. 73, No. 6, 672. https://doi.org/10.1088/0031-8949/73/6/023

[10] Jang. Two-point boundary value problems by the extended Adomian decomposition method. Journal of Computational and Applied Mathematics, 2008, Vol. 219, No. 1, 253-262. https://doi.org/10.1016/j.cam.2007.07.036

[11] M. Ghoreishi, A. M. Ismail, N. H. M. Ali. Adomian decomposition method (ADM) for nonlinear wave-like equations with variable coefficient. Applied Mathematical Sciences, 2010, Vol. 4, No. 49, 2431-2444. http://m-hikari.com/ams/ams-2010/ams-49-52-2010/ghorei shiAMS49-52-2010.pdf

[12] T. Achouri, K. Omrani. Numerical solutions for the damped generalized regularized long-wave equation with a variable coefficient by Adomian decomposition method. Communications in Nonlinear Science and Numerical Simulation, 2009, Vol. 14, No. 5, 2025-2033. https://doi.org/10.1016/j.cnsns.2008.07.011

[13] M. Wazwaz, S. M. El-Sayed. A new modification of the Adomian decomposition method for linear and nonlinear operators. Applied Mathematics and computation, 2001, Vol. 122, No. 3, 393-405. https://doi.org/10.1016/S0096-3 003(00)00060-6

[14] J. Fadaei. Application of Laplace-Adomian decomposition method on linear and nonlinear system of PDEs. Applied Mathematical Sciences, 2011, Vol. 5, No. 27, 1307-1315. http://m-hikari.com/ams/ams-2011/ams-25-28-2011/fadaei AMS25-28-2011.pdf

[15] Jang. Exact solutions to one dimensional non-homogeneous parabolic problems by the homogeneous Adomian decomposition method. Applied mathematics and computation, 2007, Vol. 186, No. 2, 969-979. https://doi.org/10.1016/j.amc.2006.08.044

[16] Cheniguel, A. Ayadi. Solving non homogeneous heat equations by the Adomian decomposition method. In International Mathematical Forum, 2011, Vol. 6, No. 13, 639-649. http://dx.doi.org/10.12988/imf.2014.371

[17] M. Wazwaz. The combined Laplace transform-Adomian decomposition method for handling nonlinear Volterra integro-differential equations. Applied Mathematics and Computation, 2010, Vol. 216, No. 4, 1304-1309. https://doi.org/10.1016/j.amc.2010.02.023

[18] Z. H. Khan, R. Gul, W. A. Khan. Application of Adomian decomposition method for sumudu transform. NUST Journal of Engineering Sciences, 2008, Vol. 1, No. 1, 40-44. https://doi.org/10.24949/njes.v1i1.25

[19] J. Biazar, E. Babolian, R. Islam. Solution of the system of ordinary differential equations by Adomian decomposition 
method. Applied mathematics and Computation, 2004, Vol. 147, No. 3, 713-719.https://doi.org/10.1016/S0096-3003(0 2)00806-8

[20] T. M. Elzaki, S. A. Alkhateeb. Modification of Sumudu transform "Elzaki transform" and Adomian decomposition method. Applied Mathematical Sciences, 2015, Vol. 9, No. 13, 603-611. http://dx.doi.org/10.12988/ams.2015.411968

[21] R. Khandelwal, Y. Khandelwal. Solution of Blasius Equation Concerning with Mohand Transform. International Journal of Applied and Computational Mathematics, 2020, Vol. 6, No. 5, 1-9. https://doi.org/10.1 007/s40819-020-00871-w

[22] R. I. Nuruddeen, L. Muhammad, A. M. Nass, T. A. Sulaiman. A review of the integral transforms-based decomposition methods and their applications in solving nonlinear PDEs. Palestine Journal of Mathematics, 2018, Vol. 7, No. 1, 262-280. https://pjm.ppu.edu/sites/default/fil es/papers/PJM_October_2017_31.pdf

[23] R. Khandelwal, P. Kumawat, Y. Khandelwal. Solution of the Blasius equation by using Adomian Kamal transform. International Journal of Applied and Computational Mathematics, 2019, Vol. 5, No. 1, 20-34. https://doi.org/10.1007/s40819-019-0601-7

[24] Ahmad. Kamal Adomian Decomposition Method for Solving Nonlinear Wave-Like Equation with Variable Coefficients. Journal of Advances in Mathematics and Computer Science, 2019, Vol. 31, No. 4, 1-11. https://doi.org/10.9734/ jamcs/2019/v31i430119

[25] R. Khandelwal, P. Kumawat, Y. Khandelwal. Kamal decomposition method and its application in solving coupled system of nonlinear PDE's. Malaya Journal Matematik, 2018, Vol. 6, No. 3, 619-625. http://www.ijfresce.org/download/conferences/ICETEAS_ 19/ICETEAS_19_Track/1549095662_02-02-2019.pdf

[26] Pushpam, C. D. Kumar. Kamal decomposition method for solving nonlinear delay differential equations. Bulletin of Pure \& Applied Sciences-Mathematics and Statistics, 2019, Vol. 38, No. 1, 231-234. http://dx.doi.org/10.5958/2320-3 226.2019.00021.3

[27] J. Ahmad, M. Ayub. Application of Kamal Decomposition Method on Integro-Differential Equations. Journal of Advanced Physics, 2017, Vol. 6, No. 4, 557-561. https://doi.org/10.1166/jap.2017.1371

[28] Kamal, H. Sedeeg. The New Integral Transform "Kamal Transform". Advances in Theoretical and Applied Mathematics, 2016, Vol. 11, No. 4, 451-458. https://www.ripublication.com/atam16/atamv11n4_14.pdf

[29] M. Mathai, H. J. Haubold. An Introduction to Fractional Calculus. New York: Nova Science Publishers, 2017.

[30] A. Opanuga, S. Edeki, H. I. Okagbue, G. Akinlabi. A novel approach for solving quadratic Riccati differential equations. International Journal of Applied Engineering Research, 2015, Vol. 10, No. 11, 29121-29126. http://eprints.covenantuniversity.edu.ng/5459/\#.YcQm0W $\mathrm{hBw} 2 \mathrm{y}$

[31] S. Odetunde, O. A. Taiwo. A decomposition algorithm for the solution of fractional quadratic Riccati differential equations with Caputo derivatives. American Journal of Computational and Applied Mathematics, 2014, Vol. 4, No. 3, 83-91. https://doi.org/10.5923/j.ajcam.20140403.03

[32] Sumiati, M. D. Johansyah, E. Rusyaman, A. K. Supriatna. Numerical method for transient solution of the fractional logistic differential equation in population growth model. In AIP Conference Proceedings, 2020, Vol. 2264, No. 1, 040004. https://doi.org/10.1063/5.0023797

[33] Sumiati, E. Rusyaman, Sukono, A. T. Bon. A Review of Adomian Decomposition Method and Applied to Deferential Equations. In Proceedings of the International Conference on Industrial Engineering and Operations Management, Pilsen, Czech Republic, July, 2019, 23-26. 